\title{
Medición de la tercera misión en las universidades públicas estatales en México por medio del análisis envolvente de datos
}

\begin{abstract}
Measurement of the Third Stream in the State Public Universities in Mexico, through the Data Envelopment Analysis
\end{abstract}

Medição da terceira missão em universidades públicas estaduais no México por meio de análise envoltória de dados

Maribel González-Cadena

Universidad Autónoma del Estado de Hidalgo, México maribel_gonzalez4257@uaeh.edu.mx https://orcid.org/0000-0001-5371-0442

Angélica María Vázquez-Rojas Universidad Autónoma del Estado de Hidalgo, México angelica_vazquez4048@uaeh.edu.mx https://orcid.org/0000-0003-2907-5383

\section{Resumen}

La tercera misión de las instituciones de educación superior busca generar y aplicar conocimiento que coadyuve al impulso del bienestar social a nivel regional, nacional e internacional. Para lograrlo, las IES deben ayudar a la construcción de proyectos innovadores (científicos y tecnológicos) que permitan desarrollar metodologías disciplinarias y multidisciplinarias que faciliten la resolución de los problemas reales que tiene la sociedad. A más de diez años de esta misión actual, es importante contar con un sistema de indicadores que ayuden a medir su eficiencia con la finalidad de conocer la situación que guardan las universidades públicas estatales (UPE) en México en relación con dicha misión. Por lo tanto, el objetivo de esta investigación es identificar el grado de avance de las dimensiones de emprendimiento, innovación y compromiso social mediante la medición de la eficiencia 


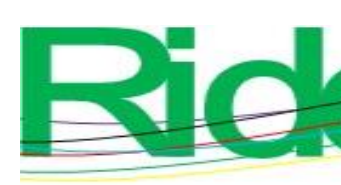

Revista Iberoamericana para la Investigación y el Desarrollo Educativo ISSN $2007-7467$

\section{Resumo}

A terceira missão das instituiçõos de ensino superior visa gerar e aplicar conhecimentos que contribuam para a promoção do bem-estar social a nível regional, nacional e internacional. Para tal, as IES devem contribuir para a construção de projetos inovadores (científicos e tecnológicos) que permitam o desenvolvimento de metodologias disciplinares e multidisciplinares que facilitem a resolução de problemas reais que a sociedade apresenta. Mais de dez anos depois desta missão atual, é importante contar com um sistema de indicadores que ajude a medir sua eficiência para conhecer a situação das universidades públicas estaduais (UPE) no México em relação a essa missão. Portanto, o objetivo desta pesquisa é identificar o grau de avanço das dimensões empreendedorismo, inovação e compromisso social por meio da medição da eficiência técnica. Como hipótese, propõe-se que a eficiência das UPEs no México em relação à sua terceira missão seja superior a $70 \%$. Utilizando a metodologia de análise envoltória de dados (DEA), foram obtidos os seguintes resultados: a eficiência técnica média com retornos variáveis de escala foi de 91,30\%. A dimensão inovação foi a que obteve o maior número de universidades com eficiência, enquanto a dimensão empreendedorismo obteve a UPE menos eficiente. Isso significa que as instituições realizam atividades destinadas a promover sua terceira missão.

Palavras-chave: análise envoltória de dados, compromisso social, eficiência, empreendedorismo, inovação.

Fecha Recepción: Junio 2020

Fecha Aceptación: Noviembre 2020

\section{Introducción}

La tercera misión de las instituciones de educación superior (IES) busca apoyar el desarrollo social y económico que se fundamenta en la responsabilidad social y en su compromiso de transformar el conocimiento en valor económico por medio de actividades claves, como generar conocimiento aplicable e impulsar la innovación, formar profesionistas que asesoren proyectos de $\mathrm{I}+\mathrm{D}+\mathrm{i}$ y participar en proyectos con emprendedores y en colaboración con el resto de agentes económicos (Vilalta, 2013).

A partir de estas actividades, Bueno y Casani (2007) proponen tres direcciones para identificar las líneas de acción que ayudan a cumplir la tercera misión de las universidades. La primera está fundamentada en el emprendimiento como creador de valor, riqueza y 


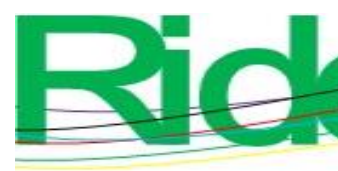
Revista Iberoamericana para la
Investigación y el Desarrollo Educativo
ISSN $2007-7467$

empleo; la segunda, basada en la transferencia del conocimiento (innovación), y la última es la extensión de sus actividades hacia el desarrollo económico y social de su comunidad (compromiso social).

A más de diez años de su fundación en las IES a nivel mundial, Bueno y Casani (2007) señalan que es importante medir y cuantificar las actividades relacionadas con la tercera misión para conocer sus avances y generar nuevas estrategias. Sin embargo, también manifiestan que cada institución debe contar con un sistema de indicadores debido a las características propias de cada universidad.

En este contexto, las IES en México deben conocer el avance que ha tenido su tercera misión con la finalidad de identificar tanto las dimensiones eficientes como las que requieren trabajo.

\section{Revisión de la literatura}

La tercera misión de la IES tiene sus cimientos en el desarrollo de estrategias para "transferencia de conocimiento a la sociedad, sustentada en la innovación, el compromiso social y el emprendimiento; promoviendo la medición y evaluación de rendimiento sobre la generación, uso, aplicación y explotación del conocimiento con los actores externos y la sociedad" (Secundo, Pérez, Martinaitis y Leither, 2017, p. 229).

Las actividades que comprenden dicha misión en los países europeos son emprendimiento, innovación y compromiso social, mientras que en Latinoamérica la consideran como extensión, es decir, una forma de difundir la cultura y el deporte (CalderónMartínez, 2017; Ruiz, 2004). Sin embargo, para Marulanda y Rojas (2019), independientemente del país donde se localice la universidad, esta misión incorpora diversas actividades que no son tomadas en cuenta dentro de la primera y segunda misión, esto es, educación continua, acceso público a conferencias y bienes culturales, trabajo voluntariado y consultoría, entre otros.

Esta variedad de actividades que integran la tercera misión ha originado que existan diferentes metodologías y criterios para comparar su desarrollo en distintas IES. Aun así, existen asociaciones de profesionistas en transferencia de tecnología que aplican diferentes sistemas de indicadores para cuantificar y conocer la situación que guardan las universidades respecto a su tercera misión (Bueno y Casani, 2007). 


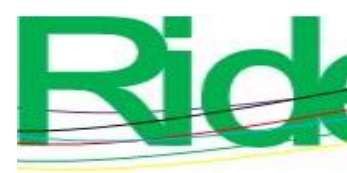

Revista Iberoamericana para la Investigación y el Desarrollo Educativo ISSN 2007 - 7467

En este sentido, De la Torre (2016) menciona que un sistema de indicadores propios ayudaría a describir, monitorear, estudiar y evaluar las actividades de la tercera misión en las universidades. De este modo, se podría redefinir la organización de colaboradores y objetivos, así como conocer sus capacidades, rendimiento obtenido, compromiso con la región y probabilidad de transferencia de conocimiento.

Por su parte, Astigarraga y Eizagirre (2017) señalan que el impulso de la tercera misión contribuiría al desarrollo económico y social, por lo que sugieren varias conceptualizaciones y actividades con diferentes finalidades. Desde el punto de vista tradicional, se consideran actividades de vinculación con prestación de servicios (comercialización de tecnología). Esto origina que se cuantifiquen los resultados económicos obtenidos por medio de la transferencia, número de empresas creadas basadas en los resultados de la investigación, contratos realizados con empresas (por asesorías y proyectos conjuntos) y patentes obtenidas. Es decir, se toman en cuenta los resultados que permiten impulsar la economía local y regional.

Desde el punto de vista de la vinculación universidad-empresa, los indicadores están orientados a medir la calidad e intensidad de esta relación para crear nuevas funciones universitarias que permitan mejorarla. En tal sentido, se considera la incorporación de estudiantes de doctorado y doctores que realicen investigación directa con las empresas. No obstante, Astigarraga y Eizagirre (2017) comentan que "las relaciones entre universidadempresa no solamente son en aspectos productivos, sino que incluye actividades que ayuden a comprender y organizar las interacciones entre el conocimiento y la vida comunitaria” (p. 76).

Asimismo, existen diversos conjuntos de indicadores para una sola dimensión. Autores como Bensing, Caris-Verhallen, Dekker, Delnoij y Groenewegen (2003) y Piva y Rossi-Lamastra (2013) advierten que contar con demasiados indicadores dificulta su medición, ya que se considerarían diferentes objetivos o se orientarían al desarrollo de la tercera misión en un contexto muy específico. Por eso, y para procurar la homogenización de los indicadores, existen tres sistemas utilizados en diversas universidades europeas para intentar conocer el progreso que han tenido las IES en relación con dicha misión.

El primer sistema es el Science and technology policy research at the universityt (SPRU proyect) de Molas-Gallart, Salter, Patel, Scott y Duran (2002). Estos autores diseñaron una metodología que incluye doce categorías para evaluar tanto actividades como 


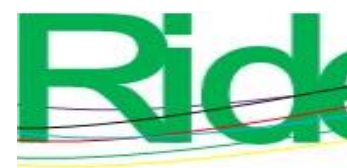

Revista Iberoamericana para la Investigación y el Desarrollo Educativo ISSN $2007-7467$

capacidades. Otro sistema es el proyecto Policies for research and innovation in the movey (PRIME) del Observatory of European University (OEU), creado con el objetivo de desarrollar estudios enfocados en las universidades para realizar evaluaciones comparativas científicas, análisis de patentes y relaciones entre la universidad y la industria (PRIME, 2006). Finalmente, se encuentra el European indicators and ranking methodology for University Third Mission; su finalidad es fomentar y mejorar la contribución a la sociedad por parte de las IES, mejorar la calidad, eficiencia y eficacia de los sistemas educativos en Europa, potenciar la excelencia y mejorar la visibilidad de las actividades universitarias orientadas a servir a la sociedad y al sector empresarial (European Indicators and Ranking Methodology for University Third Mission [E3M], 2012).

Estos, por supuesto, no son los únicos sistemas usados para cuantificar la tercera misión en las IES; sin embargo, no consideran la dimensión de compromiso social debido a que las universidades no suelen exhibir los resultados obtenidos o las actividades relacionadas con dicha dimensión.

A pesar de que estos sistemas ya se han empleados en diferentes IES europeas y latinoamericanas para conocer la situación que guardan con su tercera misión, es necesario que las universidades mexicanas apliquen alguno de esos, ya que están comprometidas con la participación en el desarrollo económico y social de nuestro país por medio de la elaboración de diferentes proyectos.

\section{Planteamiento del problema}

Tradicionalmente, la evaluación de la eficiencia de la educación superior se ha realizado por medio del cálculo de indicadores de calidad, como matrícula, egreso, deserción escolar, entre otros. No obstante, esta estimación se ha acrecentado en los últimos años debido a la rendición de cuentas y el compromiso de informar sobre el uso de recursos otorgados por el erario. Como consecuencia, se han instaurado diferentes criterios que permiten medir la eficiencia de las IES. Por ejemplo, la determinación de la eficiencia a través de indicadores relacionados con la transferencia del conocimiento, el cálculo de actividades relacionadas exclusivamente con la primera y segunda misión, y — en menor cantidad — la medición de la eficiencia mediante acciones de las tres misiones.

En cualquiera de los criterios antes mencionados, se utilizan inputs que son modificadas o usadas durante la actividad para generar una salida u output, con el objetivo 


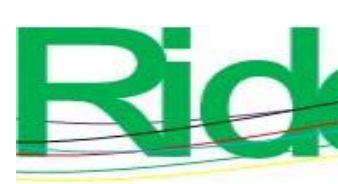

Revista Iberoamericana para la Investigación y el Desarrollo Educativo ISSN $2007-7467$

de proveer información para la toma de decisiones (Cuenca, Li, Boza, Alarcón y Lario, 2008). Como consecuencia de la medición de la eficiencia, en países europeos se han establecido políticas públicas para las IES que ha llevado a recortes presupuestarios, contratación de personal de tiempo parcial y aumento del financiamiento privado. En cambio, en nuestro país, el gobierno mexicano - por medio de sus diferentes programas sectoriales de educaciónha establecido políticas públicas que están orientadas al aseguramiento de la calidad de la educación superior, disminuir la diferencia y diversidad de instituciones que imparten este tipo de educación (evaluación y acreditación de programas educativos, programas de formación y mejoramiento del profesorado y aumento de la matrícula, entre otros) (Secretaría de Educación Pública [SEP], 2013).

Sin embargo, se debe resaltar la escasez de estudios empíricos sobre dicho tema (Calderón-Martínez, 2017; Pedroza y Ortiz, 2013) debido a la carencia de políticas públicas que coadyuven a conocer el progreso que han tenido las IES mexicanas en relación con las actividades de la tercera misión y debido a que no se cuenta con un sistema de indicadores apropiado sobre las características de estas universidades, lo cual ayudaría a conocer el grado de eficiencia y, sobre todo, si las instituciones desean cumplir con los nuevos desafíos planteados en torno a la educación superior a nivel mundial.

Explicado lo anterior, en el presente estudio se ha formulado la siguiente pregunta de investigación: ¿cuál es el grado de avance de la tercera misión, medido en términos de eficiencia técnica, de las Universidades Públicas Estatales (UPE) mexicanas?

\section{Justificación}

Esta investigación trata de contribuir a la valoración de la tercera misión de las UPE en México. La razón de este propósito se halla en que las referidas instituciones deben conocer los resultados de las actividades desarrolladas en torno a esa misión, lo cual serviría para tomar decisiones que contribuirían al desarrollo económico y social de la región aledaña.

Además, se propone un sistema de indicadores que se adapte a nuestra realidad mexicana basado en el SPRU proyect, ya que - de acuerdo con De la Torre (2016) - dicho sistema se orienta a las actividades en sí, y no a los resultados ni al impacto que han tenido en la sociedad. Además de este sistema de indicadores, para la dimensión del compromiso social se considera la política institucional focalizada en la atención a las necesidades y expectativas sociales de la Asociación Nacional de Universidades e Instituciones de 


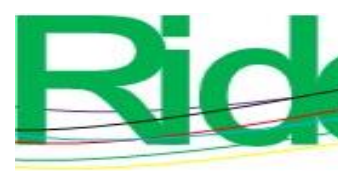

Revista Iberoamericana para la
Investigación y el Desarrollo Educativo
ISSN $2007-7467$

Educación Superior (Anuies) (2016), como una forma de adecuar las tareas de esta dimensión a lo que se encuentran haciendo las UPE actualmente.

Por lo tanto, en esta investigación se presenta un análisis de la eficiencia técnica de las UPE desde la perspectiva metodológica del análisis envolvente de datos (DEA, por sus siglas en inglés).

\section{Objetivo general}

Identificar el grado de avance de las actividades de la tercera misión en las UPE con la finalidad de contribuir a la mejora de la calidad y competitividad de la educación superior en México mediante un análisis de la eficiencia técnica.

\section{Hipótesis}

Para cumplir con el objetivo planteado en este trabajo se procede a desarrollar la siguiente hipótesis:

- La eficiencia de las universidades públicas estatales en México con respecto a su tercera misión es mayor a $70 \%$.

\section{Metodología}

Esta investigación se sustenta en un diseño no experimental de tipo transversal con alcance descriptivo y comparativo.

\section{Propuesta y selección de las unidades de análisis e indicadores}

La SEP clasifica a las IES en diversas preferencias de formación con base en los intereses y objetivos profesionales que los estudiantes desean conseguir. El Sistema de Educación Superior Pública (SESP) está integrado por siete subsistemas, de los cuales las UPE son las que tienen el mayor número de alumnos, docentes y profesores investigadores que pertenecen al Sistema Nacional de Investigadores (SNI); además, ocupa el segundo lugar en cuanto al financiamiento otorgado por el Estado. Aunado a esto, el $100 \%$ de dichas instituciones están afiliadas a la Asociación Nacional de Universidades e Instituciones de Educación Superior (Anuies), y en menor proporción al Consorcio de Universidades Mexicanas (CUMex), organismos que tienen como objetivo el aseguramiento de la calidad en los programas educativos y el desarrollo de funciones de docencia, investigación y 


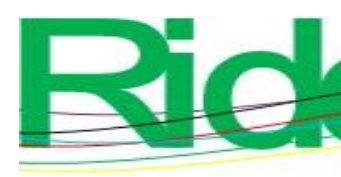

Revista Iberoamericana para la Investigación y el Desarrollo Educativo ISSN $2007-7467$

extensión. Por lo anterior, las unidades de análisis para este trabajo son las 34 UPE que integran dicho grupo de IES.

\section{Concepto de eficiencia y su medición}

Según Rodríguez (2009), la eficiencia es "el aprovechamiento de todas las oportunidades posibles para mejorar la situación de algunas personas sin que la situación de otras empeore" (p. 44). De acuerdo con Farrell (1957), las unidades de decisión (DMU, por sus siglas en inglés, decision making unit) están formadas por dos elementos: la eficiencia técnica (TE), que es cuando la empresa obtiene el máximo de outputs con la combinación de inputs empleados (Cachanosky, 2012), y la eficiencia asignativa (AE) o capacidad de los productores para combinar inputs y outputs de la mejor manera, considerando los precios y productos marginales (Vázquez-Rojas, 2011). Este análisis se enfoca en la eficiencia técnica porque muestra si los recursos de las UPE son explotados al máximo de su capacidad productiva.

La eficiencia técnica se puede valorar por medio de la orientación de los inputs o de los outputs. La orientación a los inputs $\left(\mathrm{ET}_{1}\right)$, minimiza el uso de estos para un nivel dado de outputs. Y con orientación a los outputs $\left(\mathrm{ET}_{\mathrm{o}}\right)$ maximiza los outputs para un nivel dado de inputs (Vázquez-Rojas, 2011). Debido al mínimo grado de control que tienen las UPE en relación con sus inputs, es conveniente tratar de maximizar los outputs, sin tratar de disminuir los inputs con que cuenta cada universidad. Por lo anterior, se considera la orientación al output como una forma de evaluar su eficiencia de las UPE en relación con las actividades de la tercera misión.

Por su parte, Álvarez (2013) señala que la función de la frontera es el límite de producción, y se considera como el punto de referencia para calcular la ineficiencia. Dicha frontera está compuesta por funciones de producción, costo y beneficio. En cuanto a las fronteras de producción, se dividen con base en la técnica empleada para su construcción, que puede ser por medio de técnicas paramétricas y de técnicas no paramétricas. Para Rodríguez (2009) la ventaja de esta última es que la medición de la eficiencia se lleva a cabo mediante la comparación de cada DMU, a través de una combinación lineal que permite obtener indicadores para cada una de las unidades, como el DEA. 


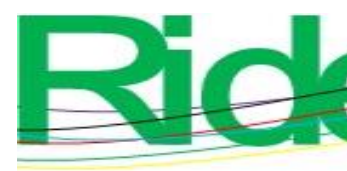

Revista Iberoamericana para la Investigación y el Desarrollo Educativo ISSN $2007-7467$

\section{Medición de la eficiencia en la educación superior a través del DEA}

La técnica DEA fue creada por Charnes, Cooper y Rhodes (1978) como una herramienta metodológica para el cálculo de la eficiencia técnica de las DMU por medio de un programa matemático de optimización. El criterio que emplea esta técnica para identificar la frontera de producción como una envolvente a los datos que considere todas aquellas unidades eficientes y el conjunto de unidades hipotéticas construidas a partir de estas aplicando el supuesto de convexidad. La frontera resultante se considera factible y eficiente, quedando el resto de unidades (ineficientes) por debajo de ella. Asimismo, la medida de eficiencia obtenida por esta técnica es relativa, ya que a cada unidad se le confronta con aquellas que operan con un valor similar de inputs y outputs con la finalidad de diagnosticar su situación en la envolvente (eficientes) o para distinguir sus unidades de referencias con la finalidad de cambiar las ineficiencias por eficiencias (Vázquez-Rojas, 2011).

La eficiencia, para el DEA, es el resultado del cociente de único output sobre un único input; si el resultado es 1 , se dice que la DMU es eficiente, mientras que aquellas que obtenga < 1 se consideran ineficientes. En consecuencia, se puede comparar cuáles unidades de análisis son "mejores" y con cuáles se debe trabajar para serlo.

En el sector educativo, el DEA ha sido utilizado para analizar su eficiencia. Autores como Becerril-Torres, Álvarez-Ayuso y Nava-Rogel (2012), Martí, Puertas y Calafat (2014), De Witte y López-Torres (2015), De la Torre (2016) y Villarreal y Tohmé (2017), entre otros, la han aplicado en diferentes IES del mundo para estimar la frontera de producción a partir de una serie de indicadores.

Para aplicar el DEA en este análisis, es necesario seleccionar alguno de los dos modelos básicos empleados: el primero es el Modelo de rendimiento constante a escala (CCR) — propuesto por Charnes et al. (1978)—, el cual asume un rendimiento de escala constante y aporta medidas de eficiencia proporcional sin considerar implicaciones organizativas, tales como el tamaño de las instituciones o el nivel de producción. Y el Modelo de rendimiento a escala (BCC) — creado por Banker, Charnes y Cooper (1984) — asume rendimientos variables a escala. Su objetivo es evaluar la eficiencia técnica quitando la influencia de las economías de escala en la evaluación de la eficiencia de las DMU. Es decir, dicho modelo considera la posibilidad de existencia de ineficiencias derivadas de las diferencias entre las escalas operativas en cada DMU (Fuentes-Pascual, 2011). 


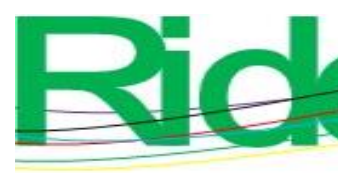

Revista Iberoamericana para la Investigación y el Desarrollo Educativo ISSN 2007 - 7467

En cuanto a la selección del modelo básico del DEA, de acuerdo con Avkiran (2001), para la selección del tipo de rendimiento que se desee utilizar en una investigación, se debe medir la eficiencia técnica aplicando el CCR y el BCC modelos con la finalidad de conocer si existen diferencias entre ellos. En el caso que se obtuvieran resultados con diferentes DMU eficientes, se aplica el modelo BCC; en caso de no encontrar diferencia entre los resultados, se utiliza el modelo CCR. Para el cálculo de la eficiencia técnica se utiliza el software DEAP 2.1 (Coelli, 1996). Una vez aplicado, se obtiene que para el modelo CCR se tienen diez UPE con puntajes menores a 1.0, mientras que en el modelo BCC, nueve UPE presentaron ineficiencia. Por lo tanto, aunque es una diferencia muy pequeña, se opta por el modelo BCC.

\section{Selección de inputs y outputs}

Para esta investigación, se estiman como inputs las actividades de la primera y segunda misión (matrícula, profesores de tiempo completo-investigador y subsidio en educación). En la tabla 1 se presentan dichos indicadores con sus definiciones.

Tabla 1. Selección de inputs y sus definiciones

\begin{tabular}{|c|l|}
\hline Inputs & \multicolumn{1}{|c|}{ Definición } \\
\hline Matrícula & $\begin{array}{l}\text { Conjunto de alumnos inscritos durante un } \\
\text { ciclo escolar en una institución o plantel } \\
\text { educativo (SEP, 2008). }\end{array}$ \\
\hline Profesores de tiempo completo & $\begin{array}{l}\text { Término que abarca a todas las personas } \\
\text { (investigadores, analistas y auxiliares o } \\
\text { asistentes de investigación) que participan en } \\
\text { las tareas propias de un proyecto de } \\
\text { investigación, es decir, en las actividades } \\
\text { teóricas, metodológicas y prácticas (SEP, } \\
\text { 2008). }\end{array}$ \\
\hline Subsidio en educación & $\begin{array}{l}\text { Asignación que otorga el gobierno federal o } \\
\text { el estatal a las IES para el cumplimiento de } \\
\text { los fines de estas (SEP, 2008). }\end{array}$ \\
\hline
\end{tabular}

Fuente: Elaboración propia

Por otra parte, los outputs se dividen en las tres dimensiones que integran la tercera misión. En las siguientes tablas se exhiben los productos empleados por cada una de ellas; es decir, en la tabla 2 outputs para las actividades de emprendimiento; en la tabla 3 outputs que 


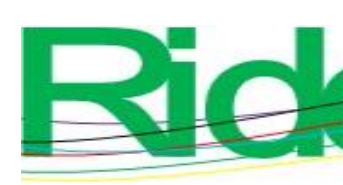

Revista Iberoamericana para la

Investigación y el Desarrollo Educativo

ISSN 2007 - 7467

se consideran para la dimensión de innovación; finalmente, en la tabla 4 outputs para la dimensión de compromiso social.

Tabla 2. Outputs empleados para la dimensión emprendimiento

\begin{tabular}{|c|c|c|}
\hline Dimensión & Output & Definición \\
\hline \multirow{9}{*}{ : } & $\begin{array}{l}\text { Patentes } \\
\text { concedidas } \\
\text { Patentes solicitadas }\end{array}$ & $\begin{array}{l}\text { Derecho exclusivo que se concede sobre una invención. En } \\
\text { términos generales, una patente faculta a su titular a decidir si } \\
\text { la invención puede ser utilizada por terceros y, en ese caso, de } \\
\text { qué forma. Como contrapartida de ese derecho, en el } \\
\text { documento de patente publicado, el titular de la patente pone } \\
\text { a disposición del público la información técnica relativa a la } \\
\text { invención (OMPI, 2018). }\end{array}$ \\
\hline & Empresa incubada & $\begin{array}{l}\text { Empresas que son apoyadas por las IES a los nuevos } \\
\text { emprendedores, asesorando y prestando la infraestructura } \\
\text { para disminuir riesgos de costos de marcha y el proceso } \\
\text { natural de aprendizaje (Wompner, 2007). }\end{array}$ \\
\hline & Marcas & $\begin{array}{l}\text { Todo signo susceptible de representación gráfica, capaz de } \\
\text { distinguir en el mercado; productos, servicios o } \\
\text { establecimiento comerciales o industriales (INAPI, s. f.) }\end{array}$ \\
\hline & Diseño industriales & $\begin{array}{l}\text { Derecho exclusivo de explotación concedido por el Estado, a } \\
\text { través del Instituto Mexicano de la Propiedad Industrial a la } \\
\text { persona que realiza un diseño industrial y/o al titular de este; } \\
\text { es decir, al inventor/diseñador o titular del mismo (INAPI, } \\
\text { 2018). }\end{array}$ \\
\hline & Derecho de autor & $\begin{array}{l}\text { Reconocimiento que otorga el Estado a todo creador de obras } \\
\text { literarias y artísticas, en virtud del cual el autor goza de } \\
\text { derechos de tipo personal, llamado derecho moral, y } \\
\text { económico llamado derecho patrimonial (INDAUTOR, s. f). }\end{array}$ \\
\hline & Modelo de utilidad & $\begin{array}{l}\text { Títulos de propiedad industrial que, al igual que las patentes, } \\
\text { protegen invenciones, pero de escaso valor creativo o de } \\
\text { innovación no radical (INAPI, s. f.). }\end{array}$ \\
\hline & $\begin{array}{c}\text { Empleados de } \\
\text { empresas incubadas }\end{array}$ & $\begin{array}{l}\text { Persona que generalmente precisa de cierta cualificación que } \\
\text { realiza una actividad laboral por cuenta de un particular, una } \\
\text { empresa incubada o el Estado y por la que recibe una } \\
\text { contraprestación económica (Economía } 48 \text {, s. f.). }\end{array}$ \\
\hline & $\begin{array}{c}\text { Fondo para } \\
\text { empresas incubadas }\end{array}$ & $\begin{array}{l}\text { Es un fondo que tiene como objeto incentivar el crecimiento } \\
\text { económico nacional, regional y sectorial, mediante el } \\
\text { fomento a la productividad e innovación (INADEM, 2018). }\end{array}$ \\
\hline & Asesorías & $\begin{array}{l}\text { Servicio profesional que orienta al directivo de una } \\
\text { organización. Es una guía de expertos que responde dudas } \\
\text { específicas, resuelve problemas concretos y apoya a las } \\
\text { organizaciones en los trámites necesarios para su operación } \\
\text { (CENAPYME, 2019a). }\end{array}$ \\
\hline
\end{tabular}




\begin{tabular}{|c|c|}
\hline & $\begin{array}{l}\text { Revista Iberoamericana para la } \\
\text { Investigación y el Desarrollo Educativo } \\
\text { ISSN } 2007-7467\end{array}$ \\
\hline Consultoría & $\begin{array}{l}\text { Servicio profesional especializado que puede ayudar a la } \\
\text { dirección general de las organizaciones a enfrentar } \\
\text { situaciones que impiden la operación plena del negocio } \\
\text { (CENAPYME, 2019b). }\end{array}$ \\
\hline $\begin{array}{l}\text { Usuarios que } \\
\text { hicieron uso de las } \\
\text { instalaciones }\end{array}$ & $\begin{array}{l}\text { Insumos requeridos para llevar acabo los procesos que tienen } \\
\text { lugar en las instituciones escolares, lo que a su vez impacta } \\
\text { en los productos del sistema educativo (INEE, 2007). }\end{array}$ \\
\hline Eventos culturales & $\begin{array}{l}\text { Son eventos organizados y coordinados por la Unidad } \\
\text { Académica Extra programáticas y buscan, a través de la } \\
\text { puesta en escena de una variedad gama de manifestaciones } \\
\text { artísticas, esparcimiento y la recreación cultural de los } \\
\text { estudiantes, la comunidad universitaria y público en general } \\
\text { (Universidad de Concepción, s. f.) }\end{array}$ \\
\hline Eventos artísticos & $\begin{array}{l}\text { Son actividades de formación, difusión, artísticas, lúdicas y } \\
\text { recreativas (IMEP, 2018) }\end{array}$ \\
\hline $\begin{array}{l}\text { Proyectos con } \\
\text { financiamiento } \\
\text { externo y Número } \\
\text { de proyectos } \\
\text { financiados por } \\
\text { Conacyt o por } \\
\text { estancias externas }\end{array}$ & $\begin{array}{l}\text { Procedimiento científico que tiene como objetivo buscar } \\
\text { información y formular hipótesis sobre un fenómeno social o } \\
\text { científico (Tamayo, 1999). }\end{array}$ \\
\hline
\end{tabular}

Fuente: Elaboración propia 
Tabla 3. Outputs empleados para la dimensión innovación

\begin{tabular}{|c|c|c|}
\hline Dimensión & Output & Definición \\
\hline \multirow{9}{*}{ 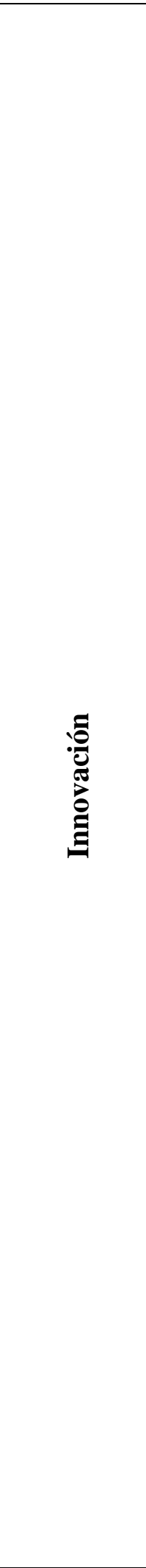 } & $\begin{array}{l}\text { Artículos } \\
\text { publicados en } \\
\text { SCOPUS }\end{array}$ & $\begin{array}{l}\text { Es un medio por el cual se difunde la generación de } \\
\text { conocimiento; se logra mediante la investigación de los } \\
\text { investigadores en las IES (Miyahira, 2017). }\end{array}$ \\
\hline & $\begin{array}{l}\text { Número de } \\
\text { organizaciones que } \\
\text { participan en } \\
\text { proyectos de } \\
\text { investigación }\end{array}$ & $\begin{array}{l}\text { Propuesta de investigación en la que se describen la fase de } \\
\text { planificación (conceptual y de diseño) de la investigación que } \\
\text { se va a iniciar (Icart, Fuente Isaz y Pulpón, 2001). }\end{array}$ \\
\hline & $\begin{array}{lr}\text { Alumnos } & \text { que } \\
\text { participan } & \text { en } \\
\text { estancias } & \text { de } \\
\text { investigación } & \\
\text { saliente } & \\
\end{array}$ & \multirow[t]{2}{*}{$\begin{array}{l}\text { Desplazamiento de estudiantes, docentes, investigadores o } \\
\text { administrativos hacia otra institución para realizar semestres } \\
\text { académicos, pasantías y prácticas, investigaciones, o para } \\
\text { asistir o participar en programas de educación continua } \\
\text { (Universidad de San Buenaventura, 2018). }\end{array}$} \\
\hline & $\begin{array}{c}\text { Movilidad } \\
\text { académica saliente }\end{array}$ & \\
\hline & $\begin{array}{l}\text { Cursos de } \\
\text { educación continua } \\
\text { (sin considerar } \\
\text { cursos de idiomas) }\end{array}$ & $\begin{array}{l}\text { Programas de formación y actualización (presenciales o en } \\
\text { línea) enfocados a mejorar e impulsar el camino profesional y } \\
\text { experiencia de vida, de manera práctica, competitiva y } \\
\text { socialmente responsable (UNID, 2019). }\end{array}$ \\
\hline & $\begin{array}{l}\text { Posgrados que } \\
\text { pertenecen al PNPC }\end{array}$ & $\begin{array}{l}\text { El Programa Nacional de Posgrados de Calidad (PNPC) forma } \\
\text { parte de la política pública de fomento a la calidad del } \\
\text { posgrado nacional que el Conacyt y la Subsecretaría de } \\
\text { Educación Pública han impulsado de manera } \\
\text { ininterrumpidamente desde } 1991 \text { (Conacyt, 2019). }\end{array}$ \\
\hline & $\begin{array}{l}\text { Egresados } \\
\text { búsqueda } \\
\text { empleo }\end{array}$ & $\begin{array}{l}\text { Recién egresado de una IES que busca conseguir un empleo } \\
\text { dentro de su área de formación durante los primeros } 6 \text { meses } \\
\text { de haber concluido sus estudios (Domínguez, Silva, Castorena, } \\
\text { Barrera y Ramírez, 2017). }\end{array}$ \\
\hline & $\begin{array}{l}\text { Satisfacción de } \\
\text { egresados }\end{array}$ & $\begin{array}{l}\text { Encuesta que permite conocer la opinión en términos de } \\
\text { satisfacción de los egresados acerca de diferentes aspectos } \\
\text { (UCAM, 2015) }\end{array}$ \\
\hline & $\begin{array}{l}\text { Convenios de } \\
\text { colaboración en el } \\
\text { último año }\end{array}$ & $\begin{array}{l}\text { Vínculos de colaboración con empresas y organismos públicos } \\
\text { o privados relacionados al sector productivo, con el objetivo } \\
\text { de transferir tecnologías desarrolladas en las IES y, formar } \\
\text { alianzas estratégicas con empresas por medio de proyectos de } \\
\text { I+D+i, a fin de apoyar su desarrollo económico y competitivo } \\
\text { (UAM, 2019). }\end{array}$ \\
\hline
\end{tabular}




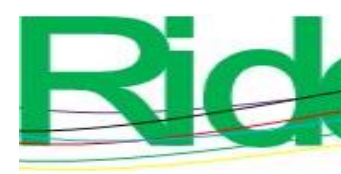

Revista Iberoamericana para la Investigación y el Desarrollo Educativo ISSN $2007-7467$

\section{Determinación de la frecuencia}

Para la obtención de la información de los indicadores seleccionados, se realizó una revisión documental de planes de desarrollo institucional, informes rectorales, anuarios estadísticos y la base de datos del EXECUM (UNAM, 2017), de las cuales solo se encontraron resultados para las 31 universidades que proporcionaron la información de los indicadores propuestos en esta investigación (es decir, se contó con $91.17 \%$ de participación).

Por su número de alumnos, las instituciones se clasifican en mega, grandes y pequeñas (Galaz, 1998). Derivado de dicha clasificación, se considera pertinente calcular la frecuencia de las actividades que llevan a cabo esas instituciones con la finalidad de contar con aquellas que realizan el mismo número de actividades, sin importar su tamaño. En la tabla 5, se exhibe el resultado del cálculo de frecuencia (relativa y acumulada). Una vez calculada, solo se consideran las UPE que realizan entre 18 y 26 tareas, porque en este rango se encuentra un mayor número de universidades; de ese modo se incluye a la mayoría y se determina su eficiencia en relación con la tercera misión.

Tabla 5. Cálculo de frecuencia

\begin{tabular}{|c|c|c|c|c|}
\hline Rangos & f1 & F1 & h1 & H1 \\
\hline 0 & 0 & 0 & 0 & 0 \\
\hline 3 & 0 & 0 & 0 & 0 \\
\hline 6 & 0 & 0 & 0 & 0 \\
\hline 9 & 0 & 0 & 0 & 0 \\
\hline 12 & 3 & 3 & 0.967742 & 0.096742 \\
\hline 15 & 3 & 6 & 0.0967742 & 0.1935484 \\
\hline 18 & 4 & 10 & 0.1290323 & 0.3225806 \\
\hline 21 & 11 & 21 & 0.3548387 & 0.6774194 \\
\hline 24 & 7 & 28 & 0.2258065 & 0.09032258 \\
\hline 27 & 3 & 31 & 0.0967742 & 1.00 \\
\hline Total & $\mathbf{3 1}$ & & $\mathbf{1 . 0 0}$ & \\
\hline
\end{tabular}

Fuente: Elaboración propia

Las 23 UPE que se encuentran en dicho rango son las siguientes: Universidad de Guadalajara, Universidad de Guanajuato, Universidad de Sonora, Universidad Veracruzana, Benemérita Universidad Autónoma de Puebla, Universidad Autónoma de Baja California, Universidad Autónoma de Nuevo León, Universidad Autónoma de Sinaloa; Universidad Autónoma de Chihuahua, Universidad Autónoma de Tamaulipas, Universidad Autónoma de 


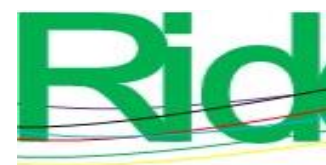

둔

Revista Iberoamericana para la

Investigación y el Desarrollo Educativo

ISSN 2007 - 7467

Querétaro, Universidad Autónoma de Chiapas, Universidad Autónoma de San Luis Potosí, Universidad Autónoma de Zacatecas, Universidad Autónoma de Aguascalientes, Universidad Autónoma de Baja California Sur y Universidad Autónoma de Campeche, Universidad Autónoma de Yucatán y Universidad Autónoma de Ciudad Juárez, Universidad Autónoma del Estado de Hidalgo y Universidad Autónoma del Estado de Morelos, Universidad Juárez Autónoma de Tabasco e Instituto Tecnológico de Sonora.

\section{Resultados}

Derivado de la obtención de resultados sobre la eficiencia técnica de cada UPE, en el sentido de rendimientos variables a escala, se observa que 21 universidades cuentan con mejores prácticas, es decir, $91.30 \%$ de las instituciones se encuentran dentro de la frontera de producción, al obtener un puntaje de 1.0 en su eficiencia de escala (tabla 6).

Tabla 6. Eficiencia técnica sentido BCC

\begin{tabular}{|c|c|c|}
\hline UPE & VRSTE & Escala \\
\hline UDG & 1.00 & 1.00 \\
\hline UABC & 1.00 & 1.00 \\
\hline UANL & 1.00 & 1.00 \\
\hline UV & 1.00 & 1.00 \\
\hline BUAP & 1.00 & 1.00 \\
\hline UACH & 1.00 & 1.00 \\
\hline UAEH & 1.00 & 1.00 \\
\hline UAEM & 1.00 & 1.00 \\
\hline UJAT & 1.00 & 1.00 \\
\hline UAQ & 1.00 & 1.00 \\
\hline UACJ & 1.00 & 1.00 \\
\hline UGTO & 1.00 & 1.00 \\
\hline UNISON & 1.00 & 1.00 \\
\hline UNACH & 1.00 & 1.00 \\
\hline UASLP & 1.00 & 1.00 \\
\hline UAZ & 1.00 & 1.00 \\
\hline ITSON & 1.00 & 1.00 \\
\hline UAA & 1.00 & 1.00 \\
\hline UADY & 1.00 & 1.00 \\
\hline UABS & 1.00 & 1.00 \\
\hline UACAM & 1.00 & 1.00 \\
\hline
\end{tabular}

Fuente: DEAP 2.1 Coelli (1996) y elaboración propia 

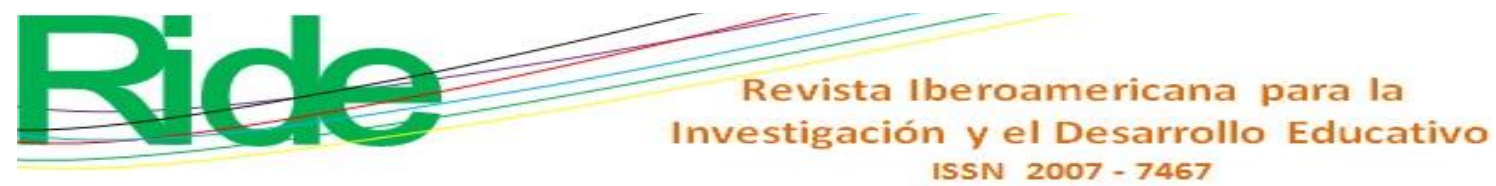

Para identificar si la ineficiencia de las DMU se debe a que están operando bajo rendimientos decrecientes de escala (DRS, por sus siglas en inglés, Diminishing Returns to Scale) - es decir, cuando la cantidad utilizada de inputs se modifican y como consecuencia la cantidad de outputs disminuye- o por rendimientos crecientes de escala (IRS, por sus siglas en inglés Increasing Return to Scale) —es decir, cuando se modifica la cantidad utilizada en los inputs, entonces la cantidad de outputs aumenta- En la tabla 7 se puede observar a la Universidad Autónoma de Sinaloa que se encuentra en el segmento de rendimientos decrecientes de escala (DRS), lo que significa que al aumentar sus inputs, sus outputs no aumentan en la misma proporción.

Tabla 7. UPE con rendimiento decreciente de escala (DRS)

\begin{tabular}{|c|c|c|}
\hline UPE & VRSTE & Escala \\
\hline UAS & 0.948 & 0.948 \\
\hline
\end{tabular}

Fuente: Elaboración propia

En la tabla 8 se exhibe a la Universidad Autónoma de Tamaulipas (UAT), que se encuentra en el segmento de rendimientos crecientes de escala (IRS); como consecuencia, al aumentar sus inputs, el incremento de sus outputs será mayor que la proporción adicional.

Tabla 8. UPE con rendimiento creciente de escala (IRS)

\begin{tabular}{|c|c|c|}
\hline UPE & VRSTE & Escala \\
\hline UAT & 0.524 & 0.997 \\
\hline
\end{tabular}

Fuente: Elaboración propia.

En estos primeros resultados se enseña a las UPE eficientes en relación con las actividades de la tercera misión. Sin embargo, para dar respuesta al objetivo planteado, se aplica nuevamente el DEA, pero por dimensiones separadas con la finalidad de reafirmar su liderazgo o identificar la dimensión que deben trabajar más en ella para fomentar el desarrollo de dicha misión. 


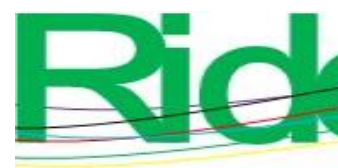

Revista Iberoamericana para la
Investigación y el Desarrollo Educativo
ISSN $2007-7467$

\section{Dimensión de emprendimiento}

Para medir la eficiencia de las UPE bajo la dimensión del emprendimiento se considera el mismo número de inputs; sin embargo, para los outputs solo se consideran ocho variables, las cuales son patentes (solicitadas y concedidas), marcas, diseños industriales y modelos de utilidad, empresas incubadas y número de empleados en empresas incubadas, fondo para empresas incubadas, número de usuarios que utilizaron las instalaciones y asistencia a eventos culturales. En la tabla 9 se aprecian las UPE eficientes en emprendimiento con sentido VRS con orientación al output.

Tabla 9. UPE eficientes en la dimensión de emprendimiento

\begin{tabular}{|c|c|c|}
\hline UPE & VRSTE & Escala \\
\hline UDG & 1.00 & 1.00 \\
\hline UABC & 1.00 & 1.00 \\
\hline UACJ & 1.00 & 1.00 \\
\hline UGTO & 1.00 & 1.00 \\
\hline UNISON & 1.00 & 1.00 \\
\hline UASLP & 1.00 & 1.00 \\
\hline ITSON & 1.00 & 1.00 \\
\hline UAA & 1.00 & 1.00 \\
\hline UADY & 1.00 & 1.00 \\
\hline
\end{tabular}

Fuente: Elaboración propia

Como se observa, al considerar únicamente los outputs de emprendimiento, solo nueve UPE continúan siendo eficientes, es decir, $60.87 \%$ del total de universidades se considera ineficientes.

En relación con las UPE que se encuentran por debajo de la frontera producción con rendimientos decrecientes de escala (DRS), son tres universidades las que al incrementar sus inputs, su producción no aumentará en la misma proporción. En la tabla 10 se visualizan dichas IES con su puntaje de eficiencia de escala que obtuvieron para la dimensión de emprendimiento.

Tabla 10. UPE con DRS en la dimensión de emprendimiento

\begin{tabular}{|c|c|c|}
\hline UPE & VRSTE & Escala \\
\hline UANL & 0.879 & 0.879 \\
\hline UV & 0.397 & 0592 \\
\hline BUAP & 0.853 & 0.853 \\
\hline
\end{tabular}

Fuente: Elaboración propia 

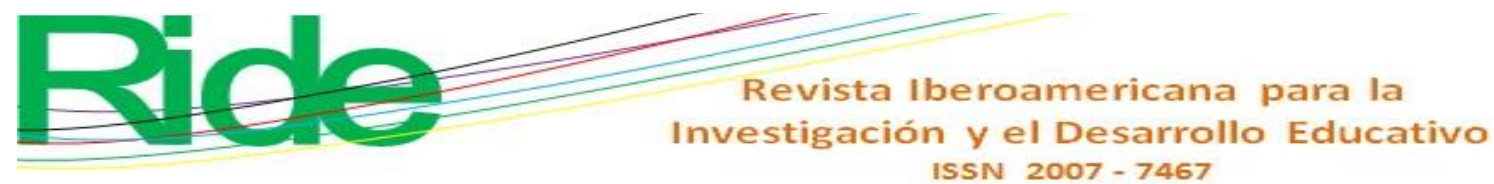

En cuanto a las UPE que presentan ineficiencia con rendimientos crecientes de escala, representan $47.83 \%$ del total de universidades. En la tabla 11 se pueden observar estas instituciones.

Tabla 11. UPE con IRS para la dimensión de emprendimiento

\begin{tabular}{|c|c|c|}
\hline UPE & VRSTE & Escala \\
\hline UACH & 0.945 & 0.961 \\
\hline UAEH & 0.323 & 0.622 \\
\hline UJAT & 0.502 & 0.810 \\
\hline UAT & 0.209 & 0.744 \\
\hline UAQ & 0.617 & 0.777 \\
\hline UNACH & 0.631 & 0.732 \\
\hline UAZ & 0.312 & 0.652 \\
\hline UABS & 0.547 & 0.547 \\
\hline UACAM & 0.782 & 0.782 \\
\hline
\end{tabular}

Fuente: Elaboración propia

\section{Dimensión de innovación}

Para esta dimensión, los outputs que ayudan a medir la eficiencia son monto de proyectos financiados por Conacyt, número de proyectos de investigación, artículos publicados (ISI y SCOPUS), número de organizaciones que participan en los proyectos de investigación, estancia de investigación saliente (docentes y alumnos), docentes en movilidad saliente, cursos de educación continua, número de posgrados que pertenecen al PNPC, alumnos inscritos en programas de posgrado que pertenecen al PNPC y número de convenios firmados. Como se puede observar, esta dimensión está constituida por catorce outputs, es decir, es la dimensión de la tercera misión que tiene más producción. Al aplicar el DEA en esta categoría, se obtiene que catorce UPE son eficientes en términos de innovación, y solo $39.13 \%$ son ineficientes. En la tabla 12 se pueden visualizar las universidades eficientes. 


\section{Revista Iberoamericana para la Investigación y el Desarrollo Educativo ISSN 2007 - 7467}

Tabla 12. UPE eficientes para la dimensión de innovación

\begin{tabular}{|c|c|c|}
\hline UPE & VRSTE & Escala \\
\hline UDG & 1.00 & 1.00 \\
\hline UABC & 1.00 & 1.00 \\
\hline UV & 1.00 & 1.00 \\
\hline UACH & 1.00 & 1.00 \\
\hline UAEH & 1.00 & 1.00 \\
\hline UAEM & 1.00 & 1.00 \\
\hline UAQ & 1.00 & 1.00 \\
\hline UACJ & 1.00 & 1.00 \\
\hline UASLP & 1.00 & 1.00 \\
\hline UAZ & 1.00 & 1.00 \\
\hline ITSON & 1.00 & 1.00 \\
\hline UAA & 1.00 & 1.00 \\
\hline UABS & 1.00 & 1.00 \\
\hline UACAM & 1.00 & 1.00 \\
\hline
\end{tabular}

Fuente: Elaboración propia

Las UPE que son ineficientes en términos de rendimientos decrecientes a escala para la dimensión de innovación representan $17.39 \%$ de las veintitrés instituciones que participan en este análisis. En la tabla 13 se encuentran las UPE con su puntaje obtenido.

Tabla 13. UPE con DRS en la dimensión de innovación

\begin{tabular}{|c|c|c|}
\hline UPE & VRSTE & Escala \\
\hline UANL & 0.820 & 0.820 \\
\hline BUAP & 0.549 & 0.549 \\
\hline UNACH & 0.872 & 0.998 \\
\hline UADY & 0.917 & 0.921 \\
\hline
\end{tabular}

Fuente: Elaboración propia

La ineficiencia en términos de rendimientos crecientes a escala en la dimensión de innovación son cinco UPE que obtuvieron puntajes $<1.00$ por encontrarse por debajo de la frontera de producción. En la tabla 14 se aprecian las universidades con sus respectivos valores. 
Tabla 14. UPE con IRS para la dimensión de innovación

\begin{tabular}{|c|c|c|}
\hline UPE & VRSTE & Escala \\
\hline UAS & 0.496 & 0.952 \\
\hline UJAT & 0.626 & 0.974 \\
\hline UAT & 0.390 & 0.959 \\
\hline UGTO & 0.800 & 0.989 \\
\hline UNISON & 0.655 & 0.935 \\
\hline
\end{tabular}

Fuente: Elaboración propia

\section{Dimensión de compromiso social}

En esta etapa de la medición de la eficiencia, considerando únicamente la variables de compromiso social como outputs, se hallaron las siguientes: número de convenios en el último año, número de conferencias impartidas, número de programas de radio y televisión, número de programas de radio, actividades relacionadas con el medio ambiente, personal capacitado en responsabilidad social, número de proyectos para comunidades vulnerables, número de personas beneficiadas en situaciones vulnerables y número de servicios para atender a la comunidad vulnerable.

En relación con los resultados para esta dimensión, 12 UPE se encuentran en la frontera producción, es decir, son las universidades eficientes. Esto significa que solo $52.17 \%$ de ellas están dentro de este grupo con mejor actuación en su compromiso social. En la tabla 15 aparecen las UPE que muestran un mejor desempeño en compromiso social. 


\section{Revista Iberoamericana para la Investigación y el Desarrollo Educativo ISSN 2007 - 7467}

Tabla 15. UPE eficientes en la dimensión de compromiso social

\begin{tabular}{|c|c|c|}
\hline UPE & VRSTE & Escala \\
\hline UANL & 1.00 & 1.00 \\
\hline UV & 1.00 & 1.00 \\
\hline UACH & 1.00 & 1.00 \\
\hline UAEH & 1.00 & 1.00 \\
\hline UAEM & 1.00 & 1.00 \\
\hline UNISON & 1.00 & 1.00 \\
\hline UNACH & 1.00 & 1.00 \\
\hline UASLP & 1.00 & 1.00 \\
\hline ITSON & 1.00 & 1.00 \\
\hline UADY & 1.00 & 1.00 \\
\hline UABS & 1.00 & 1.00 \\
\hline UACAM & 1.00 & 1.00 \\
\hline
\end{tabular}

Fuente: Elaboración propia

En este escenario, se observa a tres UPE que son ineficientes en términos rendimientos decrecientes a escala, lo que significa $13.04 \%$ del total de universidades. En la tabla 16 se muestran dicho resultado.

Tabla 16. UPE con DRS en la dimensión de compromiso social

\begin{tabular}{|c|c|c|}
\hline UPEs & VRSTE & Escala \\
\hline UAS & 0.746 & 0.746 \\
\hline UJAT & 0.544 & 0.544 \\
\hline UAZ & 0.564 & 0.996 \\
\hline
\end{tabular}

Fuente: Elaboración propia

Finalmente, las UPE con eficiencia con rendimientos crecientes a escala para la dimensión de compromiso social son ocho. En la tabla 17 se pueden visualizar cuáles son las universidades y sus resultados. 
Tabla 17. UPE con IRS para la dimensión de innovación

\begin{tabular}{|c|c|c|}
\hline UPE & VRSTE & Escala \\
\hline UDG & 0.003 & 0.065 \\
\hline UABC & 0.307 & 0.894 \\
\hline BUAP & 0.122 & 0.859 \\
\hline UAT & 0.114 & 0.576 \\
\hline UAQ & 0.460 & 0.736 \\
\hline UACJ & 0.065 & 0.178 \\
\hline UGTO & 0.662 & 0.996 \\
\hline UAA & 0.629 & 0.789 \\
\hline
\end{tabular}

Fuente: Elaboración propia

\section{Discusión}

La tercera misión en las IES ayuda a las universidades a tener una participación más activa con la sociedad por medio de actividades que motivan la investigación, el desarrollo y la innovación tecnológica y social. Al mismo tiempo, esta misión promueve el cumplimiento de su "función social", lo que posibilita un mayor involucramiento en la vida social y cultural de la región o país donde se localizan.

En este sentido, la Unión Europea reconoce el nuevo papel que tienen las universidades, y como consecuencia ha establecido diferentes propuestas que coadyuven al progreso de su tercera misión, tales como incremento en la inversión de ciencia y tecnología en relación con el producto interno bruto, la vinculación más cercana con empresas, el mejoramiento de la transferencia del conocimiento (orientado al emprendimiento, innovación y cooperación social) y la evaluación de resultados (CE, 2003).

En nuestro país, las universidades han incorporado actividades relacionadas con la tercera misión como una forma de cumplir con los retos de la educación superior a nivel mundial. Sin embargo, a diferencia de las universidades europeas se carece de un sistema de indicadores adaptados a sus características que ayude a conocer el avance que han tenido en el cumplimiento de dicho fin.

En este escenario, se han realizado estudios de tipo documental en nuestro país, y los principales resultados muestran que la dimensión de emprendimiento es la que presenta mayor avance en actividades como patentes (solicitadas y otorgadas) y número de empresas incubadas (Pedroza y Ortiz, 2013), datos que coinciden con lo reportado por CalderónMartínez (2017). Aun así, los citados autores subrayan que la falta de información hace difícil la medición de dicha misión. 

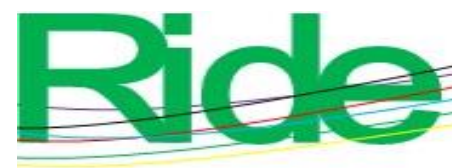

Revista Iberoamericana para la Investigación y el Desarrollo Educativo

ISSN 2007 - 7467

Igualmente, se identificaron las UPE que se hallan en el segmento de rendimientos decrecientes de escala, lo que significa que al incrementar sus insumos, su producción no aumentará en la misma proporción. Asimismo, se presentan a las universidades con rendimientos crecientes de escala, las cuales al aumentar sus inputs, obtendrán un incremento mayor en su producción. En este segmento, se localizan varias instituciones; sin embargo, la Universidad Autónoma de Tamaulipas y la Universidad Autónoma de Sinaloa tienen dicho comportamiento en las tres dimensiones.

Con la presente investigación se ha logrado reconocer que las UPE en México han incorporado a su quehacer cotidiano actividades relacionadas con su tercera misión, lo cual sirve para afirmar que la hipótesis propuesta es válida.

Finalmente, se observa que las UPE en México están fomentando la realización de las actividades que se incluyen en la tercera misión. No obstante, todavía faltan políticas públicas que impulsen el desarrollo de esta misión para que se favorezca la eficiencia en cada una de las dimensiones. De esa forma, existirá un mayor acercamiento entre estas universidades y la sociedad, lo que estimulará el desarrollo tecnológico y social de nuestro país. 


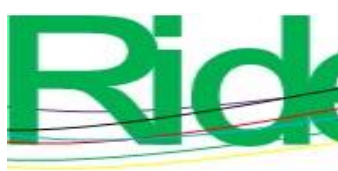

Revista Iberoamericana para la
Investigación y el Desarrollo Educativo ISSN $2007-7467$

\section{Referencias}

Álvarez, A. (2013). La medición de la eficiencia y la productividad. Ediciones Pirámide. Recuperado de https://books.google.com.mx/books?id=48uUBQAAQBAJ\&pg=PA120\&lpg=PA12 $0 \& d q=$ frontera + determinista + no + param\%C3\% A9trica\&source $=b l \& o t s=n f s 7 J l k A 4 j$ \&sig=ACfU3U1_KJQxUxdfs5L-EcdBO4PTUcgwJA\&hl=es419\&sa $=$ X\&ved $=2$ ahUKEwiF6pqWsNnkAhUROK0KHaq2AT8Q6AEwBHoECAk $\mathrm{QAQ} \# \mathrm{v}=$ onepage $\& \mathrm{q}=$ frontera $\% 20$ determinista $\% 20 \mathrm{no} \% 20$ param\%C3\%A9trica\&f $=\mathrm{t}$ rue

Ambientales, RDA y SEEDA, A. (2001). Programa y guía para la impartición del Módulo de Sensibilización Ambiental. Madrid: Red de Autoridades Ambientales. Recuperado de

https://www.cma.gva.es/areas/educacion/educacion_ambiental/educ/sensibilizacion/ pdf/MANUALDE_1.PDF

Asociación Nacional de Universidades e Instituciones de Educación Superior [Anuies] (2016). Plan de Desarrollo Institucional. Visión 2030. México. Recuperado de http://www.anuies.mx/media/docs/avisos/pdf/PlanDesarrolloVision2030.pdf

Astigarraga, E. y Eizagirre, A. (2017). El reto de la tercera misión. una visión desde Mondragón Unibertsitatea. Cuestión Universitaria, 9, 74-87. Recuperado de http://polired.upm.es/index.php/lacuestionuniversitaria/article/view/3579

Avkiran, N. (2001). Investigating technical and scale efficiencies of Australian Universities through data envelopment analysis. Socio-Economic Planning Sciences, 35(1), $57-$ 80. Doi: 10.1016/S0038-0121(00)00010-0

Baca-Tavira, N. y Herrera-Tapia, F. (2016). Proyectos sociales. Notas sobre su diseño y gestión en territorios rurales. Convergencia. Revista de Ciencias Sociales, 23(72), 6987. Recuperado de https://www.redalyc.org/pdf/105/10546932003.pdf

Banker, R. D., Charnes, A. and Cooper W. (1984). Some Models for Estimating Technical and Scale Inefficiencies in Data Envelopment Analysis. Management Science, 30(9), 1078-1092.

Retrieved

from https://personal.utdallas.edu/ ryoung/phdseminar/BCC1984.pdf

Becerril-Torres, O., Álvarez-Ayuso, I. y Nava-Rogel, R. (2012). Frontera tecnológica y eficiencia técnica de la educación superior en México. En Revista Mexicana de 


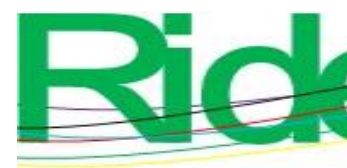

Revista Iberoamericana para la
Investigación y el Desarrollo Educativo
ISSN $2007-7467$

Investigación Educativa, 17 (54), 793-816. Recuperado de: https://www.redalyc.org/articulo.oa?id=14023127006

Bensing, J., Caris-Verhallen, W., Dekker, J. Delnoij, D. and Groenewegen, P. (2003). Doing the right thing and doing it right: Toward a framework for assessing the policy relevance of health services research. International Journal of Technology Assessment in Health Care, 19(4), 604-612. Recuperado de https://dspace.library.uu.nl/bitstream/handle/1874/21933/bensing_03_doingtherightt hing.pdf?sequence $=1$

Briz, A., Abelda, M., Fernández, J., Hidalgo, A., Pinilla, R y Pons, S. (2008). Saber hablar. Madrid: Santillana.

Bueno, E. y Casani, F. (2007). La tercera misión de la universidad: enfoques e indicadores básicos para su evaluación. Revista Economía Industrial, 366, 43-59. Recuperado de http://www.minetur.gob.es/Publicaciones/Publicacionesperiodicas/EconomiaIndustr ial/RevistaEconomiaIndustrial/366/43.pdf

Cachanosky, I. (2012). Eficiencia técnica, eficiencia económica y eficiencia dinámica. Procesos de Mercado: Revista Europea de Economía Política, 9(2), 51-80. Recuperado de http://www.hacer.org/pdf/ICachanosky00.pdf

Calderón-Martínez, M. G (2017). Tercera misión de la universidad. Una revisión de la literatura sobre emprendimiento académico. Vincula Tégica EFAN, 3(1), 364-373. Recuperado de http://www.web.facpya.uanl.mx/vinculategica/Revistas/R3/365\%20$\% 20373 \% 20-$

$\% 20$ Tercera $\% 20$ mision $\% 20$ de $\% 201$ a $\% 20$ universidad. $\% 20$ Una $\% 20$ revision $\% 20 \mathrm{de} \%$ 201a\%20literatura\%20sobre\%20emprendimiento\%20academico.pdf

Características Org. (2014). Características y estructura de la noticia periodística. Recuperado de http://caracteristicas.org/caracteristicas-noticia-periodistica/

Carta de la Tierra (s. f.). Comunidades Locales. Recuperado de https:/cartadelatierra.org/actue/comunidades-locales/

Castañares, R. L. y Cruz, E. P. (2012). Inclusión con responsabilidad social: elementos de diagnóstico y propuestas para una nueva generación de políticas de educación superior. Asociación Nacional de Universidades e Instituciones de Educación Superior. Recuperado de https://crcs.anuies.mx/wpcontent/uploads/2012/09/Inclusion-con-responsabilidad-social-ANUIES.pdf 


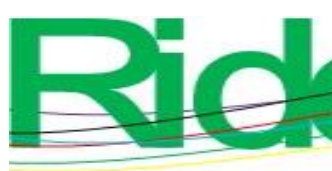

Revista Iberoamericana para la
Investigación y el Desarrollo Educativo
ISSN $2007-7467$

Centro Nacional de Apoyo a la Pequeña y Mediana Empresa [Cenapyme FCA-UNAM]. (2019a). Asesoría.

Recuperado

de http://cenapyme.fca.unam.mx/asesoria_consultoria.php

Centro Nacional de Apoyo a la Pequeña y Mediana Empresa [Cenapyme FCA-UNAM]. (2109b). Consultoría.

Recuperado de http://cenapyme.fca.unam.mx/asesoria_consultoria.php

Charnes, A., Cooper, W. and Rhodes, E. (1978). Measuring the Efficiency of Decision Making Units. European Journal of Operational Research, 2(6), 429-444. Doi: $10.1016 / 0377-2217(78) 90138-8$

Coelli, T. (1996). A guide to DEAP Version 2.1: A Data Envelopment Analysis (Computer) Program. Centre for Efficiency and Productivity Analysis, University of New England, Australia. Retrieved from http://www.owlnet.rice.edu/ econ380/DEAP.PDF

Comisión Europea [CE] (2003). Comunicado de la comisión: el papel de las universidades en la Europa del conocimiento. Recuperado de http://europa.eu.int/eurlex/es/com/cnc/2003/com2003_0058es01.pdf

Consejo Nacional de Ciencia y Tecnología [Conacyt]. (2019). Programa Nacional de Posgrados de ¿Qué es? Recuperado de https://www.conacyt.gob.mx/index.php/becas-y-posgrados/programa-nacional-deposgrados-de-calidad

Cuenca, Li., Boza, A., Alarcón, F. y Lario, F. C. (2008). Metodología para la identificación de inputs y outputs de procesos de negocio en un entorno colaborativo. Trabajo presentado en el XII Congreso de Ingeniería de Organización. Burgos, España. Recuperado de http://adingor.es/congresos/web/uploads/cio/cio2008/BUSINESS_PROCESS_MOD ELLING//395-406.pdf

De la Torre, E. (2016). Tipologías de universidades: relevancia de la tercera misión para las políticas y estrategias universitarias en el nuevo marco de la educación superior (tesis doctoral). Universidad Autónoma de Madrid, Facultad de Ciencias Económicas y Empresariales. Recuperado de https://repositorio.uam.es/handle/10486/678479 


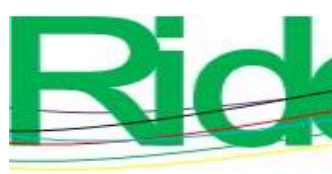

Revista Iberoamericana para la Investigación y el Desarrollo Educativo ISSN $2007-7467$

De Witte, K. and López-Torres, L. (2015). Efficiency in education. A review of literature and a way forward. Journal of the Operational Research Society, 68(4), 1-36. doi: 10.1057/jors.2015.92

Domínguez, A., Silva A., Castorena, A., Barrera, M. y Ramírez D. (2017). Investigación sobre las oportunidades de empleo para los profesionistas recién egresados utilizando BSC. En Revista Iberoamericana para la Investigación y el Desarrollo Educativo, 8 (15). doi: DOI: 10.23913/ride. v8i15.293

Donoso-Díaz, Aguirre G y Espinoza B (1999). Análisis de la eficiencia de la educación básica mediante el método de fronteras estocásticas

Economía48. (s. f.). Empleado. Recuperado de http://www.economia48.com/spa/d/empleado/empleado.htm

European Indicators and Ranking Methodology for University Third Mission [E3M]. (2012). Libro verde. El fomento y la medición de la tercera misión en las instituciones de educación superior. Doi: 10.13140/RG.2.2.25015.11687

Farrell, M. J. (1957). The measurement of productive efficiency. Journal of the royal statistical society. Series A. 120(3), 253-290. Doi: 10.2307/2343100

Fuentes-Pascual, R. (2011). Técnicas de análisis económico aplicado II. Bloque III (curso 2011-2012). Técnicas econométricas en detalle. El análisis envolvente de datos. Recuperado de https://rua.ua.es/dspace/bitstream/10045/19658/6/Materiales.Teoria.Bloque.III.pdf

Galaz, J. F. (1998). Sobre la clasificación de las instituciones mexicanas de educación superior. Revista de Educación Superior, 27(106), 1-21. Recuperado de http://publicaciones.anuies.mx/pdfs/revista/Revista106_S2A4ES.pdf

Icart, M. T., FuenteIsaz, C. y Pulpón, A. (2001). Elaboración y presentación de un proyecto de investigación y una tesina. Recuperado de http://www.publicacions.ub.edu/refs/indices/06677.pdf

Instituto Mediterráneo Estudio de Protocolo [IMEP] (2018). ¿Qué es un evento y cómo se puede clasificar? Recuperado de https://www.protocoloimep.com/articulos/que-esun-evento-y-clasificacion/

Instituto Mexicano de la Propiedad Industrial [INAPI] (2018). Diseños industriales. Recuperado de https://www.gob.mx/impi/articulos/que-son-los-disenosindustriales?idiom $=\mathrm{es}$ 


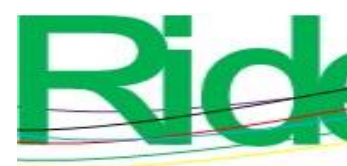

Revista Iberoamericana para la
Investigación y el Desarrollo Educativo ISSN 2007-7467

Instituto Nacional de Emprendedor [INADEM] (2018). Fondo Nacional Emprendedor. Recuperado de https://www.inadem.gob.mx/fondo-nacional-emprendedor/

Instituto Nacional de Propiedad Industrial [INAPI] (s. f.). Marca. Recuperado de https://www.inapi.cl/portal/institucional/600/w3-article-1612.html

Instituto Nacional de Propiedad Industrial [INAPI] (s. f.). Modelo de Utilidad. Recuperado de https://www.inapi.cl/portal/institucional/600/w3-article-746.html

Instituto Nacional del Derecho de Autor [INDAUTOR] (s. f.). Derechos de autor. Recuperado de http://www.indautor.gob.mx/accesibilidad/accesibilidad_autor.html Instituto Nacional para la Evaluación de la Educación [INEE] (2007). Infraestructura escolar en las primarias y secundarias de México. Recuperado de https://www.inee.edu.mx/wp-content/uploads/2019/01/infarestructuracompletoa.pdf

Martí, D., Puertas, M. y Calafat, M. (2014). Calidad y eficiencia de las universidades públicas españolas. Revista de Estudios Regionales, (99), 135-154. Recuperado de http://www.redalyc.org/pdf/755/75531857005.pdf

Marulanda, N. y Rojas, M. (2019). Ética en instituciones de educación superior para la construcción de relaciones de confianza con grupos de interés. Información Tecnológica, 30(3), 269-276. Doi: 10.4067/S0718-07642019000300269

Miyahira, J. (2017). Publicación científica: un debe ser de las instituciones de educación superior. Revista Médica Herediana, 28(2), 73-74. Doi: 10.20453/rmh. v28i2.3106

Molas-Gallart, J., Salter, A., Patel, P., Scott, A. and Duran, X. (2002). Measuring Third Stream Activities. Science and Technology Policy Research. 1-48. Retrieved from https://www.academia.edu/RegisterToDownload\#Download

Organización Mundial de la Propiedad Industrial [OMPI]. (2018). Definición de patentes. Recuperado d: http://www.wipo.int/patents/es/

Pedroza, A. y Ortiz, S. (2013). Estructura de interfaz universitaria: estudio de caso. Repositorio Institucional del ITESO. Guadalajara, Jalisco. Recuperado de http://rei.iteso.mx/bitstream/handle/11117/2154/APZ\%20-

\%20SOC\%20PROGINNT\%20_ORE\%20REV.pdf?sequence=2

Piva, E. and Rossi-Lamastra, C. (2013). Systems of indicators to evaluate the performance of university-industry alliances: a review of the literature and directions for future research. Measuring Business Excellence, 17(3), 40-54. Doi: 10.1108/MBE-01-20130004 


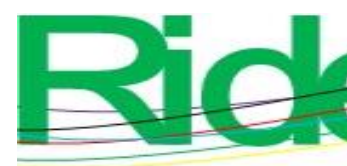

Revista Iberoamericana para la Investigación y el Desarrollo Educativo ISSN $2007-7467$

Policies for Research and Innovation in the Movey [PRIME] (2006). Methodological Guide. Observatory of the European University. 6-247. Retrieved from http://www.finhed.org/media/files/01-THIRD_MISSION_poglavlje_125_169.pdf

Rodríguez, L. G. (2009). Generación de metodología aplicando Data Envelopment Analysis (DEA), en el diseño de indicadores de eficiencia y productividad para la función de extensión de la Universidad Nacional de Colombia (tesis doctoral). Recuperado de http://bdigital.unal.edu.co/62418/

Ruiz, J. (2004). La tercera función. Revista Reencuentro, (39), 15-23. Recuperado de https://www.redalyc.org/articulo.oa?id=34003903

Secretaría de Educación Pública [SEP]. (2008). Glosario. Términos utilizados en la Dirección General de Planeación y Programación 2008. Recuperado de http://cumplimientopef.sep.gob.mx/2010/Glosario\%202008\%2024-jun-08.pdf

Secretaría de Educación Pública [SEP] (2013). Programa Sectorial de Educación 2013-2018. Recuperado de http://www.dof.gob.mx/nota_detalle_popup.php?codigo=5326569

Secretaría de Educación Pública [SEP]. (2017). Principales Cifras del Sistema Educativo Nacional 2016-2017. Recuperado de https://www.planeacion.sep.gob.mx/Doc/estadistica_e_indicadores/principales_cifra s/principales_cifras_2016_2017_bolsillo.pdf

Secundo, G. Pérez, S., Martinaitis, Z. and Leitner, K. (2017) An Intellectual Capital framework to measure universities' third mission activities. Technological Forecasting and Social Change, 1-11. Doi 10.1016/j.techfore.2016.12.013

Tamayo, M. (1999). Módulo 5. El proyecto de investigación. Instituto Colombiano para el fomento de la educación superior. Recuperado de https://www.usbcali.edu.co/sites/default/files/documentodeconsultacomplementarioel_proyecto_de_investigacion.pdf

Universidad Autónoma Metropolitana [UAM]. (2019). Coordinación general para el fortalecimiento académico y vinculación. Recuperado de http://www.vinculacion.uam.mx/index.php/universidad-y-empresa/vinculacion-conempresas/formatos-de-convenios-y-contratos

Universidad Católica San Antonio de Murcia [UCAM]. (2015). Informe de resultados Encuesta de Satisfacción de Egresados. Título de máster en Enfermería de Urgencia, Emergencias $y \quad$ Cuidados Especiales. Recuperado de 


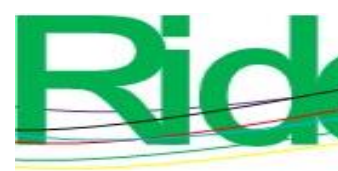

Revista Iberoamericana para la
Investigación y el Desarrollo Educativo
ISSN $2007-7467$

https://www.ucam.edu/sites/default/files/estudios/grados/gradoenfermeria/egresados_master_enfermeria_de_urgencias_15-16_y_respuesta.pdf

Universidad de Concepción (s. f.). Actividades artístico-culturales y recreativas. Recuperado de https://www.udec.cl/dise/node/704

Universidad de San Buenaventura (2018). ¿Qué es movilidad académica? Recuperado de https://www.usbmed.edu.co/internacionalizacion/movilidad-academica/que-e

Universidad Interamericana para el Desarrollo [UNID] (2019). Educación continua. Recuperado de https://www.unid.edu.mx/formacion-continua/

Universidad Nacional Autónoma de México [UNAM] (2017). Estudio comparativo de las universidades mexicanas explorador de datos. Explorador de datos UNAM. Recuperado de http://www.execum.unam.mx/\#

Universidad Nacional Autónoma de México [UNAM]. (2019). Dirección General de Cooperación $\quad$ Internacionalización. Recuperado de https://www.unaminternacional.unam.mx/es/alumno/licenciatura/movilidad-alextranjero/investigacion

Vázquez-Rojas, M. A. (2011). Eficiencia técnica y cambio de productividad en la educación superior pública: un estudio aplicado al caso español (2000-2009) (tesis doctoral). Recuperado de https://repositorio.uam.es/handle/10486/11162

Vilalta, J. (2013). La tercera misión universitaria. Innovación y transferencia de conocimientos en las universidades españolas. Studia XXI, (4), 7-9. Recuperado de http://www.studiaxxi.com/site/wp-content/uploads/00.-Cuaderno-de-trabajo-4print.pdf

Villarreal, F. y Tohmé, F. (2017). Análisis envolvente de datos. Un caso de estudio para una universidad argentina. Estudios Gerenciales, 33(144), 302-308. Doi: 10.1016/j.estger.2017.06.004

Wompner, F. (2007). Un modelo de incubadora de negocios universitaria; en la ruta de la innovación organizacional. Observatorio de la Economía Latinoamericana, 87. Recuperado de http://www.eumed.net/cursecon/ecolat/cl/2007/fw-incub.htm 


\begin{tabular}{|l|l|}
\hline Rol de contribución & Autor(es) \\
\hline Conceptualización & Maribel (principal) Angélica (igual) \\
\hline Metodología & Maribel (principal) Angélica (igual) \\
\hline Software & Maribel \\
\hline Validación & Angélica (principal) Maribel (igual) \\
\hline Análisis Formal & Maribel (principal) Angélica (igual) \\
\hline Investigación & Maribel (principal) Angélica (igual) \\
\hline Recursos & Maribel (principal) \\
\hline Curación de datos & Maribel (principal) Angélica (igual \\
\hline Escritura-preparación del borrador & Maribel \\
\hline original & \\
\hline Escritura-revisión y edición & Angélica \\
\hline Visualización & Maribel (principal) Angélica (igual) \\
\hline Supervisión & Angélica \\
\hline Administración de proyectos & Maribel \\
\hline Adquisición de fondos & Maribel (principal) Angélica (igual) \\
\hline
\end{tabular}

